\title{
On the generalized Helmholtz conditions for Lagrangian systems with dissipative forces
}

\author{
M. Crampin, T. Mestdag and W. Sarlet \\ Department of Mathematics, Ghent University \\ Krijgslaan 281, B-9000 Ghent, Belgium
}

\begin{abstract}
In two recent papers necessary and sufficient conditions for a given system of second-order ordinary differential equations to be of Lagrangian form with additional dissipative forces were derived. We point out that these conditions are not independent and prove a stronger result accordingly.
\end{abstract}

Keywords. Lagrangian systems, dissipative forces, inverse problem, Helmholtz conditions.

MSC (2000). 70H03, 70F $17,49 \mathrm{~N} 45$

\section{Introduction}

The Helmholtz conditions, for the purposes of this paper, are the necessary and sufficient conditions for a given system of second-order ordinary differential equations $f_{a}(\ddot{q}, \dot{q}, q, t)=$ 0 to be of Euler-Lagrange type, that is, for there to exist a Lagrangian $\Lambda(\dot{q}, q, t)$ such that

$$
f_{a}=\frac{d}{d t}\left(\frac{\partial \Lambda}{\partial \dot{q}^{a}}\right)-\frac{\partial \Lambda}{\partial q^{a}}
$$

Here $q^{a}$ are the generalized coordinates (collectively abbreviated to $q$ ), $\dot{q}^{a}$ the corresponding generalized velocities, and so on. We shall state the conditions shortly. The Lagrangian is supposed to be of first order, that is, independent of $\ddot{q}$ and higher-order derivative (or more properly jet) coordinates.

In two recent papers the problem of finding analogous necessary and sufficient conditions for a given set of functions $f_{a}(\ddot{q}, \dot{q}, q, t)$ to take the more general form

$$
f_{a}=\frac{d}{d t}\left(\frac{\partial \Lambda}{\partial \dot{q}^{a}}\right)-\frac{\partial \Lambda}{\partial q^{a}}+\frac{\partial D}{\partial \dot{q}^{a}}
$$

for first-order functions $\Lambda$ (a Lagrangian) and $D$ (a dissipation function) has been discussed. We shall say that in this case the equations $f_{a}=0$ are of Lagrangian form with dissipative forces of gradient type. A set of necessary and sufficient conditions is given, in terms of standard coordinates, in the fairly recent paper [3]. In a very recent paper [2] 
a version of the conditions expressed in terms of quasi-velocities, or as the authors call them nonholonomic velocities, is obtained. We shall quote the conditions from [3] explicitly in Section 2. These conditions are described as generalized Helmholtz conditions to distinguish them from the Helmholtz conditions discussed in our opening paragraph, which may be called the classical Helmholtz conditions; these must of course comprise a special case of the generalized conditions.

The main purpose of the present paper is to point out that the generalized Helmholtz conditions as stated in [3] are not independent: in fact two of them are redundant, in that they can be derived from the remaining ones. This we show in Section 2 below. We use the same formalism as [3]. Since the version of the generalized conditions obtained in [2] is equivalent to that in [3] the same redundancy is present there as well. By taking advantage of the improvement in the formulation of the generalized Helmholtz conditions that we achieve, we are able to give a shorter and more elegant proof of their sufficiency than the one to be found in [3].

There are in fact several interesting questions raised by the two papers [2, 3], only one of which will be dealt with here. In the third and final section of our paper we give an outline of these additional points of interest, which will receive a fuller airing elsewhere.

We employ the Einstein summation convention throughout.

We end this introduction with a brief summary of the results about the classical Helmholtz conditions that we shall need.

The classical Helmholtz conditions are that the $f_{a}$ should satisfy

$$
\begin{aligned}
\frac{\partial f_{a}}{\partial \ddot{q}^{b}} & =\frac{\partial f_{b}}{\partial \ddot{q}^{a}} \\
\frac{\partial f_{a}}{\partial \dot{q}^{b}}+\frac{\partial f_{b}}{\partial \dot{q}^{a}} & =2 \frac{d}{d t}\left(\frac{\partial f_{b}}{\partial \ddot{q}^{a}}\right) \\
\frac{\partial f_{a}}{\partial q^{b}}-\frac{\partial f_{b}}{\partial q^{a}} & =\frac{1}{2} \frac{d}{d t}\left(\frac{\partial f_{a}}{\partial \dot{q}^{b}}-\frac{\partial f_{b}}{\partial \dot{q}^{a}}\right) .
\end{aligned}
$$

It is a consequence of these (and not an extra condition, as stated in [3]) that

$$
\frac{\partial^{2} f_{a}}{\partial \ddot{q}^{b} \partial \ddot{q}^{c}}=0
$$

(this follows from the vanishing of the coefficient of $\dddot{q}^{c}$ in condition (44)). Thus we may write $f_{a}=g_{a b} \ddot{q}^{b}+h_{a}$, the coefficients being of first order, with $g_{a b}=g_{b a}$ as a result of condition (3). The Helmholtz conditions can be re-expressed in terms of $g_{a b}$ (assumed to be symmetric) and $h_{a}$, when they reduce to the following three conditions:

$$
\begin{aligned}
\frac{\partial g_{a b}}{\partial \dot{q}^{c}}-\frac{\partial g_{a c}}{\partial \dot{q}^{b}} & =0 \\
\frac{\partial h_{a}}{\partial \dot{q}^{b}}+\frac{\partial h_{b}}{\partial \dot{q}^{a}} & =2 \frac{\bar{d}}{d t}\left(g_{a b}\right) \\
2\left(\frac{\partial h_{a}}{\partial q^{b}}-\frac{\partial h_{b}}{\partial q^{a}}\right) & =\frac{\bar{d}}{d t}\left(\frac{\partial h_{a}}{\partial \dot{q}^{b}}-\frac{\partial h_{b}}{\partial \dot{q}^{a}}\right),
\end{aligned}
$$


where

$$
\frac{\bar{d}}{d t}=\frac{\partial}{\partial t}+\dot{q}^{c} \frac{\partial}{\partial q^{c}}
$$

That is to say, the $f_{a}$ are the Euler-Lagrange expressions of some first-order Lagrangian if and only if $f_{a}=g_{a b} \ddot{q}^{b}+h_{a}$ for some first-order functions $g_{a b}$ and $h_{a}$ such that $g_{a b}=g_{b a}$ and (6) -(8) hold. This reformulation can be found in the book by Santilli [6], for example.

\section{The generalized Helmholtz conditions}

We next turn to the analysis of the generalized Helmholtz conditions. Following the notation of [3] we set

$$
\begin{aligned}
& r_{a b}=\frac{\partial f_{a}}{\partial q^{b}}-\frac{\partial f_{b}}{\partial q^{a}}+\frac{1}{2} \frac{d}{d t}\left(\frac{\partial f_{b}}{\partial \dot{q}^{a}}-\frac{\partial f_{a}}{\partial \dot{q}^{b}}\right) \\
& s_{a b}=\frac{1}{2}\left(\frac{\partial f_{a}}{\partial \dot{q}^{b}}+\frac{\partial f_{b}}{\partial \dot{q}^{a}}\right)-\frac{d}{d t}\left(\frac{\partial f_{b}}{\partial \ddot{q}^{a}}\right) .
\end{aligned}
$$

The generalized Helmholtz conditions as given in [3] are that $r_{a b}$ and $s_{a b}$ are of first order, and that in addition

$$
\begin{aligned}
\frac{\partial f_{a}}{\partial \ddot{q}^{b}} & =\frac{\partial f_{b}}{\partial \ddot{q}^{a}} \\
\frac{\partial s_{a b}}{\partial \dot{q}^{c}} & =\frac{\partial s_{a c}}{\partial \dot{q}^{b}} \\
\frac{\partial r_{a b}}{\partial \dot{q}^{c}} & =\frac{\partial s_{a c}}{\partial q^{b}}-\frac{\partial s_{b c}}{\partial q^{a}} \\
0 & =\frac{\partial r_{a b}}{\partial q^{c}}+\frac{\partial r_{b c}}{\partial q^{a}}+\frac{\partial r_{c a}}{\partial q^{b}} .
\end{aligned}
$$

Our main concern will be with analyzing conditions (10)-(12), which correspond to (2.3e), (2.3f) and (2.3g) of [3]; we shall show that conditions (10) and (12) are redundant, being consequences of the remaining conditions.

Our first aim is to understand exactly what it means for $r_{a b}$ and $s_{a b}$ to be of first order, bearing in mind condition (9) above. From the vanishing of the coefficient of $\dddot{q}^{c}$ in $s_{a b}$ we have

$$
\frac{\partial^{2} f_{a}}{\partial \ddot{q}^{b} \partial \ddot{q}^{c}}=0
$$

As before $f_{a}=g_{a b} \ddot{q}^{b}+h_{a}$, the coefficients being of first order and $g_{a b}$ symmetric. The coefficient of $\dddot{q}^{c}$ in $r_{a b}$ is

$$
\frac{1}{2} \frac{\partial}{\partial \ddot{q}^{c}}\left(\frac{\partial g_{b d}}{\partial \dot{q}^{a}} \ddot{q}^{d}+\frac{\partial h_{b}}{\partial \dot{q}^{a}}-\frac{\partial g_{a d}}{\partial \dot{q}^{b}} \ddot{q}^{d}-\frac{\partial h_{a}}{\partial \dot{q}^{b}}\right)
$$

whence

$$
\frac{\partial g_{b c}}{\partial \dot{q}^{a}}=\frac{\partial g_{a c}}{\partial \dot{q}^{b}}
$$


The coefficient of $\ddot{q}^{c}$ in $s_{a b}$, namely

$$
\frac{\partial g_{a c}}{\partial \dot{q}^{b}}+\frac{\partial g_{b c}}{\partial \dot{q}^{a}}-2 \frac{\partial g_{a b}}{\partial \dot{q}^{c}}
$$

vanishes as a consequence. The coefficient of $\ddot{q}^{c}$ in $r_{a b}$ is

$$
\frac{\partial g_{a c}}{\partial q^{b}}-\frac{\partial g_{b c}}{\partial q^{a}}-\frac{1}{2}\left(\frac{\partial^{2} h_{a}}{\partial \dot{q}^{b} \partial \dot{q}^{c}}-\frac{\partial^{2} h_{b}}{\partial \dot{q}^{a} \partial \dot{q}^{c}}\right)
$$

an expression which for later convenience we write as $\rho_{a b c}$; we must of course have $\rho_{a b c}=0$. The remaining terms in $r_{a b}$ and $s_{a b}$ are all of first order, and we have

$$
\begin{aligned}
& r_{a b}=\frac{\partial h_{a}}{\partial q^{b}}-\frac{\partial h_{b}}{\partial q^{a}}-\frac{1}{2} \frac{\bar{d}}{d t}\left(\frac{\partial h_{a}}{\partial \dot{q}^{b}}-\frac{\partial h_{b}}{\partial \dot{q}^{a}}\right) \\
& s_{a b}=\frac{1}{2}\left(\frac{\partial h_{a}}{\partial \dot{q}^{b}}+\frac{\partial h_{b}}{\partial \dot{q}^{a}}\right)-\frac{\bar{d}}{d t}\left(g_{a b}\right) ;
\end{aligned}
$$

compare with (77) and (8), and also with equations (2.16b) and (2.17c) of [3].

The redundancy of condition (10) is a consequence of the vanishing of $\rho_{a b c}$, as we now show. We have

$$
\frac{\partial s_{a c}}{\partial \dot{q}^{b}}=\frac{1}{2}\left(\frac{\partial^{2} h_{a}}{\partial \dot{q}^{b} \partial \dot{q}^{c}}+\frac{\partial^{2} h_{c}}{\partial \dot{q}^{a} \partial \dot{q}^{b}}\right)-\frac{\bar{d}}{d t}\left(\frac{\partial g_{a c}}{\partial \dot{q}^{b}}\right)-\frac{\partial g_{a c}}{\partial q^{b}}
$$

using the commutation relation

$$
\left[\frac{\partial}{\partial \dot{q}^{a}}, \frac{\bar{d}}{d t}\right]=\frac{\partial}{\partial q^{a}}
$$

It follows that $\partial s_{a c} / \partial \dot{q}^{b}-\partial s_{b c} / \partial \dot{q}^{a}=-\rho_{a b c}=0$. That is to say, condition (10) holds automatically as a consequence of the first-order property. Furthermore, $\rho_{a b c}=0$ is equivalent to equation (2.17b) of [3]; in other words, the redundancy of (10) is actually implicit in [3], though not apparently recognized there.

Before proceeding to consider condition (12) we turn aside to make some remarks about the classical Helmholtz conditions. The calculations just carried out are essentially the same as those which lead to the version of the classical Helmholtz conditions given in equations (6) -(8) at the end of the introduction. It is easy to see that in that case $r_{a b}=s_{a b}=0$ are necessary conditions. This observation, together with the part of the argument concerning the vanishing of the coefficients of $\dddot{q}$ and $\ddot{q}$, leads to the following 
conditions:

$$
\begin{aligned}
\frac{\partial g_{a c}}{\partial \dot{q}^{b}}+\frac{\partial g_{b c}}{\partial \dot{q}^{a}} & =2 \frac{\partial g_{a b}}{\partial \dot{q}^{c}} \\
\frac{\partial h_{a}}{\partial \dot{q}^{b}}+\frac{\partial h_{b}}{\partial \dot{q}^{a}} & =2 \frac{\bar{d}}{d t}\left(g_{a b}\right) \\
\frac{\partial g_{a b}}{\partial \dot{q}^{c}}-\frac{\partial g_{a c}}{\partial \dot{q}^{b}} & =0 \\
\frac{\partial^{2} h_{a}}{\partial \dot{q}^{b} \partial \dot{q}^{c}}-\frac{\partial^{2} h_{b}}{\partial \dot{q}^{a} \partial \dot{q}^{c}} & =2\left(\frac{\partial g_{a c}}{\partial q^{b}}-\frac{\partial g_{b c}}{\partial q^{a}}\right) \\
2\left(\frac{\partial h_{a}}{\partial q^{b}}-\frac{\partial h_{b}}{\partial q^{a}}\right) & =\frac{\bar{d}}{d t}\left(\frac{\partial h_{a}}{\partial \dot{q}^{b}}-\frac{\partial h_{b}}{\partial \dot{q}^{a}}\right) .
\end{aligned}
$$

These are the conditions quoted in Remark 3 of Section 1 of [3]. However, it is now evident that two of them are redundant. Clearly condition (14) (which is the vanishing of the coefficient of $\ddot{q}^{c}$ in $s_{a b}$ ) follows from condition (16) and the symmetry of $g_{a b}$. Condition (17) is the condition $\rho_{a b c}=0$. The second part of the argument above, that leading to the relation $\rho_{a b c}=\partial s_{b c} / \partial \dot{q}^{a}-\partial s_{a c} / \partial \dot{q}^{b}$, shows that in the classical case condition (17) follows from the other conditions. When these two redundant conditions are removed we obtain the classical Helmholtz conditions in the form given at the end of the introduction.

These results in the classical case are actually very well known, though not apparently to the authors of [3, and have been known for a long time: they are to be found, for example, in Santilli's book of 1978 [6] (which is in fact referred to in [3]). For the sake of clarity we should point out a difference between the two cases: in the classical case condition (17) is completely redundant; in the generalized case it is not redundant, but occurs twice in the formulation of the conditions in [3], once in the requirement that $r_{a b}$ should be of first order and once as the condition $\partial s_{a b} / \partial \dot{q}^{c}=\partial s_{b c} / \partial \dot{q}^{a}$.

We now return to the generalized conditions, and prove that condition (12) follows from condition (11). It will be convenient to write condition (12) as

$$
\sum_{a, b, c} \frac{\partial r_{a b}}{\partial q^{c}}=0
$$

where $\sum_{a, b, c}$ stands for the cyclic sum over $a, b$ and $c$, here and below. As a preliminary remark, note that if $k_{a b c}$ is symmetric in $b$ and $c$ (say) then $\sum_{a, b, c} k_{a b c}=\sum_{a, b, c} k_{b a c}$. Now

$$
\frac{\partial r_{a b}}{\partial q^{c}}=\frac{\partial^{2} h_{a}}{\partial q^{b} \partial q^{c}}-\frac{\partial^{2} h_{b}}{\partial q^{a} \partial q^{c}}-\frac{1}{2} \frac{\bar{d}}{d t}\left(\frac{\partial^{2} h_{a}}{\partial q^{c} \partial \dot{q}^{b}}-\frac{\partial^{2} h_{b}}{\partial q^{c} \partial \dot{q}^{a}}\right)
$$

and so by the preliminary remark

$$
\sum_{a, b, c} \frac{\partial r_{a b}}{\partial q^{c}}=-\frac{1}{2} \frac{\bar{d}}{d t}\left(\sum_{a, b, c}\left(\frac{\partial^{2} h_{a}}{\partial q^{c} \partial \dot{q}^{b}}-\frac{\partial^{2} h_{b}}{\partial q^{c} \partial \dot{q}^{a}}\right)\right)=-\frac{1}{2} \frac{\bar{d}}{d t}\left(\sum_{a, b, c}\left(\frac{\partial^{2} h_{a}}{\partial q^{c} \partial \dot{q}^{b}}-\frac{\partial^{2} h_{a}}{\partial q^{b} \partial \dot{q}^{c}}\right)\right) .
$$


On the other hand, using the commutation relation (13) and the fact that $\partial / \partial q^{a}$ and $\bar{d} / d t$ commute it is easy to see that condition (11) leads to

$$
\frac{1}{2} \sum_{a, b, c}\left(\frac{\partial^{2} h_{a}}{\partial q^{c} \partial \dot{q}^{b}}-\frac{\partial^{2} h_{a}}{\partial q^{b} \partial \dot{q}^{c}}\right)=-\frac{\bar{d}}{d t}\left(\rho_{a b c}\right)=0 .
$$

We therefore reach the following proposition, which is stronger than the corresponding result in [3].

Proposition. The necessary and sufficient conditions for the equations $f_{a}(\ddot{q}, \dot{q}, q, t)=0$ to be of Lagrangian form with dissipative forces of gradient type as in (2) are that the functions $r_{a b}$ and $s_{a b}$ are of first order, that

$$
\frac{\partial f_{a}}{\partial \ddot{q}^{b}}=\frac{\partial f_{b}}{\partial \ddot{q}^{a}}
$$

and that

$$
\frac{\partial r_{a b}}{\partial \dot{q}^{c}}=\frac{\partial s_{a c}}{\partial q^{b}}-\frac{\partial s_{b c}}{\partial q^{a}}
$$

Just as in the classical case we can give an equivalent formulation of these conditions in terms of $g_{a b}$ and $h_{a}$. Bearing in mind that $r_{a b}$ and $s_{a b}$ being of first order are essential hypotheses, we find that the following conditions are equivalent to those given in the proposition above: $f_{a}=g_{a b} \ddot{q}^{b}+h_{a}$ with $g_{a b}$ symmetric, where $g_{a b}, h_{a}$ are of first order and further satisfy

$$
\begin{aligned}
\frac{\partial g_{a b}}{\partial \dot{q}^{c}}-\frac{\partial g_{a c}}{\partial \dot{q}^{b}} & =0 \\
\frac{\partial^{2} h_{a}}{\partial \dot{q}^{b} \partial \dot{q}^{c}}-\frac{\partial^{2} h_{b}}{\partial \dot{q}^{a} \partial \dot{q}^{c}} & =2\left(\frac{\partial g_{a c}}{\partial q^{b}}-\frac{\partial g_{b c}}{\partial q^{a}}\right) \\
\sum_{a, b, c}\left(\frac{\partial^{2} h_{a}}{\partial q^{b} \partial \dot{q}^{c}}-\frac{\partial^{2} h_{a}}{\partial q^{c} \partial \dot{q}^{b}}\right) & =0 .
\end{aligned}
$$

The first of these is one of the classical conditions. The second is the condition $\rho_{a b c}=0$, which holds in the classical case as we have shown. The third is just condition (19) above expressed in terms of $g_{a b}$ and $h_{a}$ (or as it turns out, in terms of $h_{a}$ alone), and $r_{a b}=s_{a b}=0$ in the classical case. It is evident therefore that the conditions above are indeed a generalization of those for the classical case.

We end this section by giving an alternative proof of the sufficiency of the generalized Helmholtz conditions, based on this formulation of them, which is shorter and in our view more elegant than the proof in [3] (necessity is an easy if tedious calculation).

We note first that if $g_{a b}$ is symmetric and satisfies $\partial g_{a b} / \partial \dot{q}^{c}=\partial g_{a c} / \partial \dot{q}^{b}$ then

$$
g_{a b}=\frac{\partial^{2} K}{\partial \dot{q}^{a} \partial \dot{q}^{b}}
$$


for some function $K=K(\dot{q}, q, t)$ (a well-known result, which also appears in [3]). Of course $K$ is not determined by this relation; in fact if $\Lambda=K+P_{a} \dot{q}^{a}+Q$, where $P_{a}$ and $Q$ are any functions of $q$ and $t$, then $\Lambda$ has the same Hessian as $K$ (the same $g_{a b}$, in other words). Our aim is to choose $P_{a}$ and $Q$ so that the given equations are of Lagrangian form with dissipative forces of gradient type as in (2), with Lagrangian $\Lambda$, assuming that the generalized Helmholtz conditions above hold. In fact we won't need to consider $Q$ because it can be absorbed: if $\Lambda$ is a Lagrangian and $D$ a dissipation function for some functions $f_{a}$, so are $\Lambda+Q$ and $D+\dot{q}^{a} \partial Q / \partial q^{a}$. We shall therefore take $Q=0$ below.

Let $E_{a}$ be the Euler-Lagrange expressions of $K$. Then $E_{a}=g_{a b} \ddot{q}^{b}+k_{a}$ for some firstorder $k_{a}$, by construction, so $f_{a}-E_{a}=h_{a}-k_{a}=\kappa_{a}$ say, where $\kappa_{a}$ is also of first order. Moreover, $f_{a}$ satisfies the generalized Helmholtz conditions by assumption, and $E_{a}$ does so by construction (it satisfies the classical conditions after all), whence $\kappa_{a}$ satisfies

$$
\begin{aligned}
\frac{\partial^{2} \kappa_{a}}{\partial \dot{q}^{b} \partial \dot{q}^{c}}-\frac{\partial^{2} \kappa_{b}}{\partial \dot{q}^{a} \partial \dot{q}^{c}} & =0 \\
\sum_{a, b, c}\left(\frac{\partial^{2} \kappa_{a}}{\partial q^{b} \partial \dot{q}^{c}}-\frac{\partial^{2} \kappa_{a}}{\partial q^{c} \partial \dot{q}^{b}}\right) & =0
\end{aligned}
$$

Let us set $\partial \kappa_{a} / \partial \dot{q}^{b}-\partial \kappa_{b} / \partial \dot{q}^{a}=R_{a b}$. Then by (20) $R_{a b}$ is independent of $\dot{q}$, and by (21)

$$
\sum_{a, b, c} \frac{\partial R_{a b}}{\partial q^{c}}=0 .
$$

There are therefore functions $P_{a}(q, t)$ such that

$$
R_{a b}=2\left(\frac{\partial P_{a}}{\partial q^{b}}-\frac{\partial P_{b}}{\partial q^{a}}\right)=\frac{\partial \kappa_{a}}{\partial \dot{q}^{b}}-\frac{\partial \kappa_{b}}{\partial \dot{q}^{a}}
$$

which is to say that if we set

$$
\pi_{a b}=\frac{\partial \kappa_{a}}{\partial \dot{q}^{b}}-\left(\frac{\partial P_{a}}{\partial q^{b}}-\frac{\partial P_{b}}{\partial q^{a}}\right)
$$

then $\pi_{b a}=\pi_{a b}$. Moreover,

$$
\frac{\partial \pi_{a b}}{\partial \dot{q}^{c}}=\frac{\partial^{2} \kappa_{a}}{\partial \dot{q}^{b} \partial \dot{q}^{c}}=\frac{\partial \pi_{a c}}{\partial \dot{q}^{b}} .
$$

It follows (just as is the case for $g_{a b}$ ) that there is a first-order function $D^{\prime}$ such that

$$
\pi_{a b}=\frac{\partial^{2} D^{\prime}}{\partial \dot{q}^{a} \partial \dot{q}^{b}},
$$

from which we obtain

$$
\kappa_{a}=\left(\frac{\partial P_{a}}{\partial q^{b}}-\frac{\partial P_{b}}{\partial q^{a}}\right) \dot{q}^{b}+\frac{\partial D^{\prime}}{\partial \dot{q}^{a}}+S_{a}
$$


where $S_{a}$ is independent of $\dot{q}$. Now take $\Lambda=K+P_{a} \dot{q}^{a}$. Denoting the Euler-Lagrange expressions of $K$ by $E_{a}$ as before, the Euler-Lagrange expressions for $\Lambda$ are

$$
E_{a}+\left(\frac{\partial P_{a}}{\partial q^{b}}-\frac{\partial P_{b}}{\partial q^{a}}\right) \dot{q}^{b}+\frac{\partial P_{a}}{\partial t}=E_{a}+\kappa_{a}-\frac{\partial D^{\prime}}{\partial \dot{q}^{a}}-S_{a}+\frac{\partial P_{a}}{\partial t} .
$$

Thus, putting

$$
D=D^{\prime}+\left(S_{a}-\frac{\partial P_{a}}{\partial t}\right) \dot{q}^{a}
$$

we get

$$
f_{a}=\frac{d}{d t}\left(\frac{\partial \Lambda}{\partial \dot{q}^{a}}\right)-\frac{\partial \Lambda}{\partial q^{a}}+\frac{\partial D}{\partial \dot{q}^{a}}
$$

as required.

This method of proof works equally well in the classical case. The proof is constructive, in the same sense that the one in [3] is, in either case. It is particularly well adapted to the familiar situation in which $g_{a b}$ is independent of $\dot{q}$, when one can take the kinetic energy $\frac{1}{2} g_{a b} \dot{q}^{a} \dot{q}^{b}$ for $K$.

\section{Concluding remarks}

We wish to make four remarks in conclusion.

The first remark concerns the nature of conditions (10)-(12) on the derivatives of $r_{a b}$ and $s_{a b}$, as originally expressed in [3] (that is, ignoring the question of dependence). In particular, bearing in mind the fact that $r_{a b}$ is skew in its indices, the condition

$$
\sum_{a, b, c} \frac{\partial r_{a b}}{\partial q^{c}}=0
$$

is suggestive: if perchance the $r_{a b}$ were functions of the $q$ alone this would have a natural interpretation in terms of the exterior calculus, being the condition for the 2 -form $r_{a b} d q^{a} \wedge d q^{b}$ to be closed, that is, to satisfy $d\left(r_{a b} d q^{a} \wedge d q^{b}\right)=0$. This point is made, in somewhat different terms, in [3] (and we appealed to the same general result in our proof of sufficiency of the generalized Helmholtz conditions in Section 2). The authors of [3] go on to say, however, that the condition above 'can be interpreted as the vanishing curvature of a symplectic space', which seems to us not to be entirely convincing. In fact it is possible to interpret the three conditions (10)-(12) collectively as signifying the vanishing of a certain exterior derivative of a certain 2-form on the space of coordinates $t, q, \dot{q}, \ddot{q}, \ldots$, a 2 -form whose coefficients involve both $r_{a b}$ and $s_{a b}$. This interpretation really arises from seeing the problem in the context of the so-called variational bicomplex (see [7] for a recent review).

Secondly, we contend that the problem we are dealing with should really be regarded as one about (second-order) dynamical systems. The point is that a dynamical system may be represented as a system of differential equations in many different coordinate 
formulations; the question of real interest is whether there is some representation of it which takes the form of an Euler-Lagrange system with dissipation, not just whether a given representation of it takes that form. Of course this point applies equally, mutatis mutandis, to the case in which there is no dissipation. Now the Helmholtz conditions as discussed in [2, 3], in both the classical and the generalized versions, suffer from the disadvantage that they are conditions for a given system of differential equations to be of Euler-Lagrange type. There is, however, an alternative approach to the problem which does deal with dynamical systems rather than equations, at least in the case in which the system can be expressed in normal form $\ddot{q}^{a}=F^{a}(\dot{q}, q, t)$. In this approach one asks (in the absence of dissipation) for conditions for the existence of a so-called multiplier, a non-singular matrix with elements $g_{a b}$, such that $g_{a b}\left(\ddot{q}^{b}-F^{b}\right)$ takes the Euler-Lagrange form (so that in particular when the conditions are satisfied $g_{a b}$ will be the Hessian of the Lagrangian with respect to the velocity variables). The basic idea is to put $h_{a}=-g_{a b} F^{b}$ in the conditions at the end of the introduction, and regard the results as a system of partial differential equations equations for $g_{a b}$ with $F^{a}$ known. The seminal paper in this approach is Douglas's of 1941 [1], which analyses in great detail the case of two degrees of freedom. For a recent review of developments since then see Sections 5 and 6 of [4] and references therein. One can in fact also formulate conditions on a multiplier for a second-order dynamical system, expressible in normal form, to be representable as equations of Lagrangian form with dissipative forces of gradient type; these generalize the known results for representation in Lagrangian form without dissipation in an interesting way.

The new ingredient in [2, by comparison with [3, is the expression of the generalized Helmholtz conditions in terms of quasi-velocities. As presented in the paper this is quite a long-drawn-out procedure, because in effect the conditions are rederived from scratch. Our third remark is that in principle this should be unnecessary: a truly satisfactory formulation of the conditions should be tensorial, in the sense of being independent of a choice of coordinates (and of course quasi-velocities are just a certain type of velocity coordinates). The approach described in the previous paragraph leads to conditions which have this desirable property.

Fourthly and finally, there is the question of whether generalized Helmholtz conditions can be derived for other kinds of "generalized force" terms than $\partial D / \partial \dot{q}^{a}$. One important case is that in which such a term is of gyroscopic type. We have obtained such conditions in this case, again using the approach discussed in our second remark above.

These points are discussed in full detail in a recently written paper [5].

\section{Acknowledgements}

The first author is a Guest Professor at Ghent University: he is grateful to the Department of Mathematical Physics and Astronomy at Ghent for its hospitality. The second author is a Postdoctoral Fellow of the Research Foundation - Flanders (FWO). 


\section{References}

[1] J. Douglas, Solution of the inverse problem of the calculus of variations, Trans. Amer. Math. Soc. 50, 71-128 (1941).

[2] U. Jungnickel, G. Kielau, P. Maisser and A. Müller, A generalization of the Helmholtz conditions for the existence of a first-order Lagrangian using nonholonomic velocities, Z. Angew. Math. Mech. 89, 44-53 (2009).

[3] G. Kielau and P. Maisser, A generalization of the Helmholtz conditions for the existence of a first-order Lagrangian, Z. Angew. Math. Mech. 86, 722-735 (2006).

[4] O. Krupková and G. E. Prince, Second order ordinary differential equations in jet bundles and the inverse problem of the calculus of variations, in: D. Krupka and D. J. Saunders (eds.) Handbook of Global Analysis (Elsevier, Amsterdam, 2008) pp. 837-904.

[5] T. Mestdag, W. Sarlet and M. Crampin, The inverse problem for Lagrangian systems with certain non-conservative forces, preprint, Ghent University, available at http://users.ugent.be/ tmestdag.

[6] R. M. Santilli, Foundations of Theoretical Mechanics I. The Inverse Problem in Newtonian Mechanics (Spinger, New York, 1978).

[7] R. Vitolo, Variational sequences, in: D. Krupka and D. J. Saunders (eds.), Handbook of Global Analysis (Elsevier, Amsterdam, 2008) pp. 1115-1163. 